\title{
Reaching the Nonlinear Regime of Raman Amplification of Ultrashort Laser Pulses
}

\author{
W. Cheng, Y. Avitzour, Y. Ping, and S. Suckewer* \\ Department of MAE, School of Engineering and Applied Science, Princeton University, Princeton, New Jersey 08544, USA \\ N. J. Fisch \\ Princeton Plasma Physics Laboratory \& Astrophysics Department, Princeton University, Princeton, New Jersey 08544, USA \\ M. S. Hur and J. S. Wurtele \\ Lawrence Berkeley National Laboratory \& Physics Department, University of California, Berkeley, California 94720, USA
}

(Received 3 November 2004; published 3 February 2005)

\begin{abstract}
The intensity of a subpicosecond laser pulse was amplified by a factor of up to 1000 using the Raman backscatter interaction in a $2 \mathrm{~mm}$ long gas jet plasma. The process of Raman amplification reached the nonlinear regime, with the intensity of the amplified pulse exceeding that of the pump pulse by more than an order of magnitude. Features unique to the nonlinear regime such as gain saturation, bandwidth broadening, and pulse shortening were observed. Simulation and theory are in qualitative agreement with the measurements.
\end{abstract}

PACS numbers: 52.38.Bv, 42.65.Dr, 52.50.Jm

The invention of chirped pulse amplification (CPA) [1,2] led to a tremendous increase of ultrashort laser pulse intensities to above $10^{20} \mathrm{~W} / \mathrm{cm}^{2}[3,4]$. However, such an ultrahigh intensity laser system was achieved using very large (on the order of $\sim 1 \mathrm{~m}^{2}$ ) and expensive compressor gratings [4]. A further increase of the pulse intensity using the CPA technique would require even larger gratings in order not to exceed the material damage threshold. Such a system would be very difficult to implement in universityscale laboratories.

In order to overcome the CPA material limit at ultrahigh intensities, different backscattering coupling techniques were proposed in plasma, including Compton scattering [5], resonant Raman backscattering [6], and Raman backscattering at an ionization front [7]. The experiments reported here utilize the resonant Raman mechanism [6], where a short seed laser pulse is amplified by a counterpropagating long pump pulse, with their frequencies satisfying the resonance relation, $\omega_{\text {pump }}=$ $\omega_{\text {seed }}+\omega_{p e}$ where $\omega_{\text {pump }}, \omega_{\text {seed }}$, and $\omega_{p e}$ are frequencies of pump, seed, and plasma, respectively; $\omega_{p e}=$ $\sqrt{4 \pi e^{2} n_{e} / m_{e}}, n_{e}$ is the plasma electron density, and $m_{e}$ and $e$ are mass and charge of an electron. The energy transfer from pump to seed is in proportion to their frequencies, so for $\omega_{\text {pump }} \geq 10 \omega_{p e}$, the efficiency can be as high as $90 \%$. What makes the resonant Raman backscatter regime attractive is that it is a simple resonant interaction, with the seed amplification strong enough to outrun other deleterious competing instabilities (such as modulational instability that can lead to the filamentation of the laser beam) [6] or to avoid superluminous precursor solutions [8], and with realizable highly compressed ultrashort pulse solutions [9].

The Raman backscattering (RBS) amplification can be divided into linear and nonlinear regimes. In the linear regime the pump depletion is negligible and the gain is independent of the seed intensity. The seed pulse is amplified and increased in duration due to the narrow bandwidth of the linear amplification. The nonlinear regime, the socalled $\pi$-pulse regime, is characterized by pump depletion and the simultaneous temporal compression of the amplified pulse. In this regime, the Raman amplification and compression of ultrashort pulses in a plasma allow intensities to reach $10^{20}-10^{21} \mathrm{~W} / \mathrm{cm}^{2}$ in a compact universityscale device, and unprecedented high intensity on the order of $10^{25} \mathrm{~W} / \mathrm{cm}^{2}$ in a larger system [9]. Such intensities open new frontiers in physics and should also lead to practical applications such as fast ignition for inertial fusion [10], x-ray lasers [11], or laser wake field accelerators [12].

Previous experiments demonstrated low amplifications in a short microcapillary [13] and gas jet plasmas [14]. Recently, energy amplification of close to 100 was demonstrated in a plasma created in a $2 \mathrm{~mm}$ long gas jet with effective (i.e., with proper density) plasma length $\sim 1-1.5 \mathrm{~mm}$. In that experiment [15], a 550 fs broadband seed (from an optical parametric generation and amplification system) was pumped by a counterpropagating $10 \mathrm{ps}$ Ti:sapphire laser pulse at a wavelength of $800 \mathrm{~nm}$. Only $10 \%$ of the seed spectrum $(0.8 \mu \mathrm{J})$ that satisfied the RBS resonance condition was amplified. While some temporal compression of seed pulse was observed at maximum amplification, the analysis of data did not provide clear evidence that the nonlinear regime was reached. In similar experiments, energy amplification of $\sim 100$ [16] were demonstrated just recently. Energy amplification of $\sim 10$ was also reported [17], with the result ascribed in part to Compton scattering [5], but the far greater part of the amplification, if not all of it, appears to be in the resonant regime associated with the other early experiments. 
What this Letter reports is the first demonstration of reaching the important nonlinear regime of RBS amplification, where we can expect the onset of pump depletion and significant pulse shortening. This regime is reached here with pulse intensity amplification $\sim 1000$ in a $2 \mathrm{~mm}$ long gas jet plasma. The intensity of the amplified exceeded that of the pump by more than 1 order of magnitude. Three major changes in the present experiment led to the new results: (1) Development of a narrow-band seed pulse with a Gaussian profile in space and time. (2) Improvement of the uniformity of the plasma with an effective length up to $2 \mathrm{~mm}$. (3) Installation of diagnostics for precise temporal and spatial alignment of the laser pulses in plasma.

The experimental setup is shown schematically in Fig. 1. The electron density $n_{e}$ was $1.1 \times 10^{19} \mathrm{~cm}^{-3}\left(\omega_{p e}=2 \times\right.$ $\left.10^{14} \mathrm{~s}^{-1}\right)$, which matched the difference between frequencies of the pump $\left(\omega_{\text {pump }}=2.4 \times 10^{15} \mathrm{~s}^{-1}\right)$ and the seed $\left(\omega_{\text {seed }}=2.2 \times 10^{15} \mathrm{~s}^{-1}\right)$. The plasma was created in a propane $\left(\mathrm{C}_{3} \mathrm{H}_{8}\right)$ gas jet through optical breakdown induced by a low-power Nd/YAG laser pulse (the "prepulse": wavelength $1.064 \mu \mathrm{m}$, pulse duration $6 \mathrm{~ns}$, and energy $\sim 500 \mathrm{~mJ}$ ), which was focused to a $\sim 50 \mu \mathrm{m}$ spot just above and parallel to a gas jet nozzle. The Rayleigh length of the prepulse was $\sim 2 \mathrm{~mm}$. Propane was used in order to have a large number of electrons from a single molecule so that a sufficiently high electron density could be achieved. A stretched Ti:sapphire laser beam (central wavelength $800 \mathrm{~nm}$ with a bandwidth of $11 \mathrm{~nm}$ and a pulse duration of $170 \mathrm{ps}$ ) was split into two beams. The first beam, which served as a pump pulse, was compressed by compressor $\mathrm{C} 1$ to $10 \mathrm{ps}$ and maintained the original bandwidth. The second beam was used as a pump pulse for a Raman shifter [18] (a $7 \mathrm{~cm}$ long barium nitrate crystal). The first Stokes beam, with the wavelength center at $873 \mathrm{~nm}$ and the same bandwidth as that of the pump, was generated in the crystal and compressed by the second compressor $\mathrm{C} 2$ to about

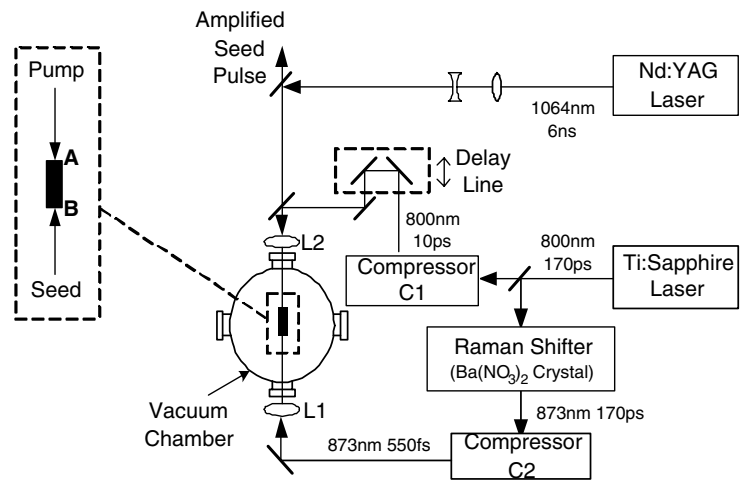

FIG. 1. Schematic of the experimental setup. Plasma is formed by the Nd:YAG laser. Part of the output from the Ti:sapphire laser is compressed by $\mathrm{C} 1$ to $10 \mathrm{ps}$ and serves as the pump. The other part passes through a Raman shifter, is compressed by $\mathrm{C} 2$ to $550 \mathrm{fs}$, and serves as the seed pulse. The two pulses are focused to the plasma in the vacuum chamber.
550 fs. The pump entered the plasma at point $A$ and the seed at point $B$. The output seed passed through a long wavelength $(>850 \mathrm{~nm})$ pass filter so that any reflected pump beam was filtered out. A small part of the amplified seed pulse was directed to a spectrometer for spectral analysis. The rest of the beam was split by a $50 \%$ beam splitter. One part was directed to a power meter and the other part was directed to an autocorrelator.

Figure 2(a) shows the output energy versus the input seed energy when the pump energy was maintained at $40 \mathrm{~mJ}$. The thermal Raman was the output signal when the seed pulse was blocked. Its energy was measured to be $\sim 80 \mu \mathrm{J}$. While the thermal Raman output was fairly stable, we observed fluctuations of the energy of the amplified pulse, possibly because the amplification process has a narrower bandwidth than the noise, and thus is more sensitive to the fluctuations of the plasma density. Only the largest five outputs (which represented the optimal conditions for amplification) from an average of 15 shots were shown in each data point. When the seed pulse was let into the plasma (the solid line), the output grew very rapidly if the seed pulse energy was less than $1.5 \mu \mathrm{J}$. As the input seed energy was increased beyond $1.5 \mu \mathrm{J}$, the growth rate decreased and the gain was saturated. For the minimum initial seed energy of $0.05 \mu \mathrm{J}$, the output was about $350 \mu \mathrm{J}$. Subtracting the thermal Raman energy (about $80 \mu \mathrm{J}$ ) from the total output, we had an energy amplification factor of $\sim 5000$. As the gain was saturated, the amplification dropped to $\sim 60$ when the input seed was at its maximum $(7.5 \mu \mathrm{J})$. The output energy versus the pump energy is shown in Fig. 2(b) (solid line) with the energy of the input seed pulse maintained at $\sim 7.5 \mu \mathrm{J}$. The dotted line shows the energy of the thermal Raman.

The pulse compression in the nonlinear regime is accompanied by bandwidth broadening. Typical spectra of the pump, the input seed and the amplified seed, are shown
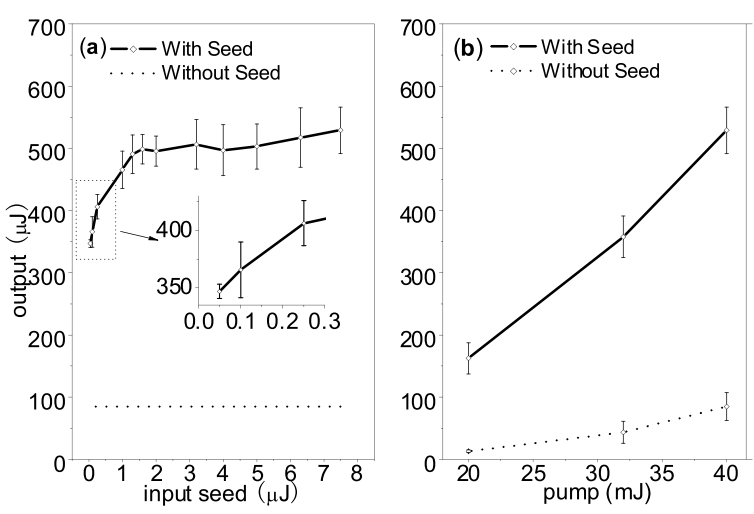

FIG. 2. Output energy versus input seed energy and pump energy. (a) Output energy versus input seed energy when the pump energy was maintained at $40 \mathrm{~mJ}$. Inset shows the output when the input was low and has the same scale as the main figure. (b) Output energy versus pump energy when the seed energy was maintained at $7.5 \mu \mathrm{J}$. In both cases, the dotted line denotes the thermal Raman. 

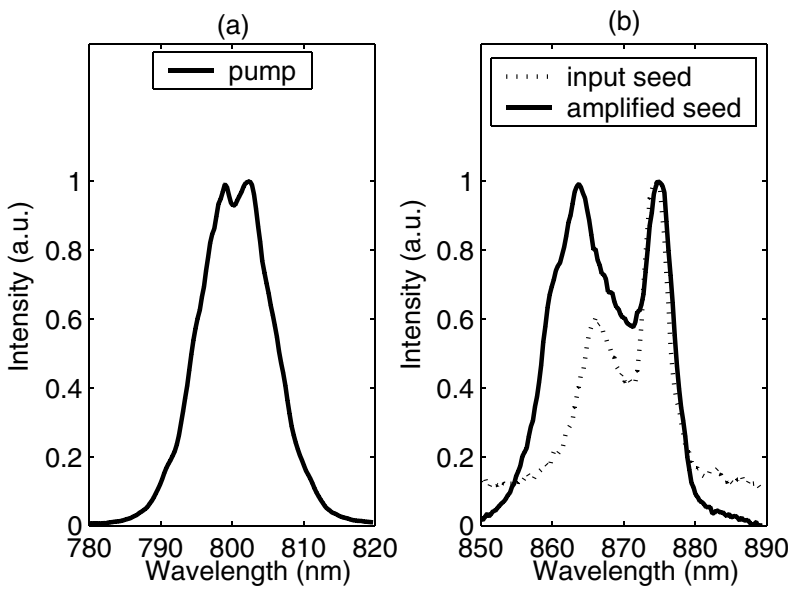

FIG. 3. Normalized (to 1) spectra of (a) pump pulse and (b) input seed pulse and amplified seed pulse for amplification of 40 .

in Fig. 3. The pump (for pumping of both the barium nitrate crystal and the plasma) and the input seed had a bandwidth of about $12 \mathrm{~nm}$. The dip in the center of the spectrum of the pump beam may have contributed to the big dip in the spectrum of the input seed pulse because of the nonlinear process of the Raman shifter [18]. The spectrum of an amplified seed pulse was measured when the energy amplification was $\sim 40$ for an initial seed energy of $7.5 \mu \mathrm{J}$. The bandwidth of the amplified seed was $\sim 19 \mathrm{~nm}$.

In order to observe the spatial profile evolution of the seed beam, it was imaged onto a CCD camera at the exit of the plasma (point $A$ in Fig. 1). In the experiment, both the pump and the seed were focused to point $B$ and had a beam size of $\sim 30 \mu \mathrm{m}$ FWHM. The seed beam profile at point $A$ in the absence of the plasma is shown in Fig. 4(a). The size of the beam was measured to be $55 \mu \mathrm{m}$ FWHM. When the plasma was present and the seed pulse was amplified by the counterpropagating pump pulse, it preserved the Gaussian profile and showed significant beam narrowing. The beam size shown in Fig. 4(b) has a FWHM of $13 \mu \mathrm{m}$. (A neutral density filter was used to reduce the amplified seed energy to avoid saturation of the CCD camera). The beam narrowing effect may be explained by the nonuniform amplification across the beam profile: the central part of the seed was amplified more due to the stronger pump in the center. The self-focusing of the amplified seed pulse in plasma may also have contributed to the beam narrowing, although we did not observe significant seed beam narrowing after it passed through the plasma without the pump pulse.

With the high amplification of the seed pulse, we were able to perform a pulse duration measurement of the amplified seed using a standard autocorrelator (Positive Light Model SSA) at various amplification stages. With the pump and seed energy maintained at $40 \mathrm{~mJ}$ and $7.5 \mu \mathrm{J}$ respectively, the energy amplification spanned a range from 10 to 60 due to the fluctuations of the plasma parameters. The pulse was observed to lengthen to a maximum of $\sim 800 \mathrm{fs}$ when the amplification was close to 10 .
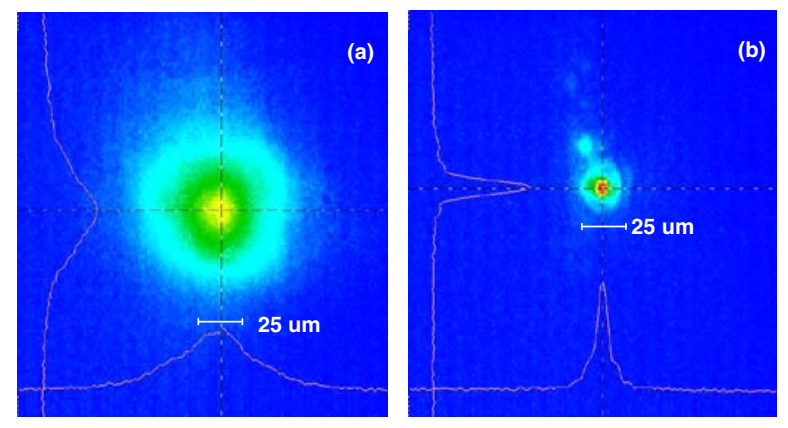

FIG. 4 (color online). Output seed pulse profiles (a) without amplification (no plasma) and (b) after amplification (image obtained through a $2.5 \%$ transmission filter).

The pulse duration began to decrease when the amplification approached 30. While the plasma fluctuations caused large standard deviations of the output pulse duration, the trend that the pulse became shorter as the amplification became higher can be seen clearly in Fig. 5 . The shortest pulse we have observed so far had a FWHM of $\sim 150 \mathrm{fs}$ when the energy amplification was $\sim 60$ with a $7.5 \mu \mathrm{J}$ input seed. At this point, the power of the seed pulse increased by more than 200 times with the intensity amplification reaching $\sim 1000$ times. The intensity of the amplified seed pulse at point $A$ in Fig. 1 was $1.7 \times$ $10^{15} \mathrm{~W} / \mathrm{cm}^{2}$. The pump pulse diameter was about $70 \mu \mathrm{m}$ FWHM at point $A$ and had an intensity of $\sim 1 \times$ $10^{14} \mathrm{~W} / \mathrm{cm}^{2}$. The intensity of the amplified seed exceeded the pump intensity at this point by more than 1 order of magnitude.

The physical parameters of the experiment are such that kinetic plasma effects influence the seed pulse amplification process. We have investigated these effects using the averaged particle-in-cell simulation code APIC [19]. The code models the electromagnetic waves in the slowly varying amplitude and phase approximation, and uses standard plasma simulation techniques to model the Langmuir wave and plasma dynamics. The seed and pump pulses are assumed to be initially Gaussian with FWHM given by the experimental values of $550 \mathrm{fs}$ and

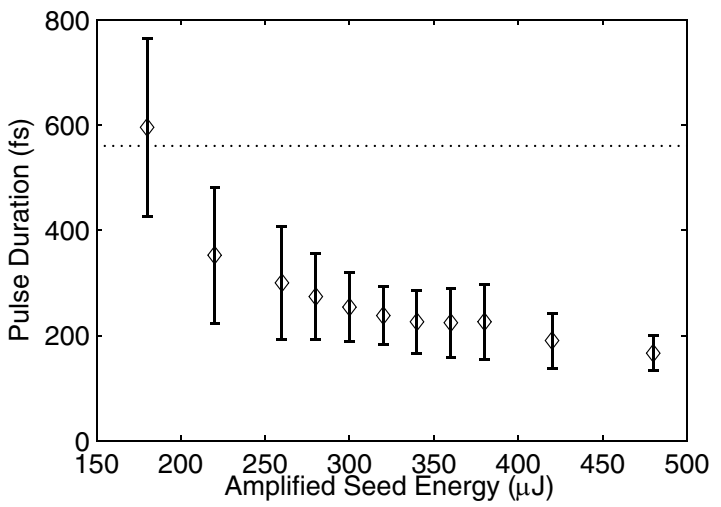

FIG. 5. The pulse duration versus energy of the amplified seed pulse. Dotted line denotes the initial pulse duration. 


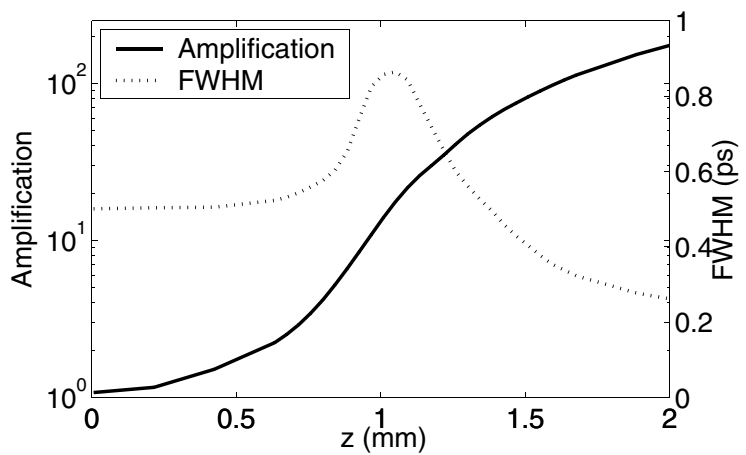

FIG. 6. The 1D, time dependent computer simulation of energy amplification (solid line) and amplified pulse duration at its FWHM (dotted line) versus plasma length for 10 ps pump.

$10 \mathrm{ps}$, respectively. The initial seed intensity was $1.6 \times$ $10^{12} \mathrm{~W} / \mathrm{cm}^{2}$, pump intensity was $1 \times 10^{14} \mathrm{~W} / \mathrm{cm}^{2}$, and both were close to experimental parameters. The plasma density was chosen so that the plasma wave (including a thermal shift) was resonant with the beat frequency of the lasers. The electrons were assumed to be initially homogeneous with a Maxwellian velocity distribution with $T_{e}=$ $50 \mathrm{eV}$, and ions were assumed to be motionless over the short time scale of the simulation. Typical simulation results are shown in Fig. 6. The seed energy amplification and the FWHM of the seed pulse duration are plotted as a function of the interaction length. The simulation clearly shows pulse stretching of the seed during the initial amplification (until roughly $1 \mathrm{~mm}$, corresponding to an energy amplification of $\sim 20$ ), followed by pulse shortening to $\sim 240 \mathrm{fs}$ at the end of $2 \mathrm{~mm}$ plasma. While the pulse duration evolution agreed quite well with the experimental measurements, the energy gain factor did not agree so well. The simulation results shown here are one-dimensional and therefore do not include the effects of diffraction and transverse variation of the laser pulses. Kinetic effects are significant when the initial value chosen for the temperature is $100 \mathrm{eV}$. At temperatures of $50 \mathrm{eV}$ and below, with Gaussian pump pulses, the qualitative features of the experiment are consistent with the experimental results.

In summary, we demonstrated energy amplification of up to $\sim 5000$ for a seed with a low input energy $(\sim 0.05 \mu \mathrm{J})$, and the amplification was reduced, by saturation effects, to $\sim 60$ for seeds with significantly higher input energy $(7.5 \mu \mathrm{J})$. The beam transverse profile of the amplified seed was narrowed by the amplification process. The measurement of the pulse duration and its spatial profile allowed us to determine a maximum amplified pulse intensity to be $\sim 1.7 \times 10^{15} \mathrm{~W} / \mathrm{cm}^{2}$, which was higher than pump intensity by more than 1 order of magnitude. This is a strong indication of reaching the nonlinear regime. The pulse was lengthened from approximately $0.5 \mathrm{ps}$ to up to $0.8 \mathrm{ps}$ when the energy amplification was $\sim 10$, and was compressed to below $\sim 200 \mathrm{fs}$ when the energy amplification reached $\sim 60$ for maximum input seed energy. This is additional evidence that the nonlinear regime was reached. We noticed, however, only a relatively small fraction of the total energy of the pump pulse was transferred to the seed pulse. Increasing the effective plasma length and pump pulse duration, together with the guiding of the pump and seed pulses through a plasma channel [20] should dramatically increase the energy transfer between the pump and the seed.

We thank A. Morozov and J.H. Kim for their assistance in the experiments, and N. Tkach for technical support. Very helpful discussions with R. Berg, V. Malkin, G. Shvets, and E. Valeo are highly appreciated. This work was supported by DARPA and NSF (PHYS) grants.

*Electronic address: suckewer@ princeton.edu

[1] D. Strickland and G. Mourou, Opt. Commun. 56, 219 (1985).

[2] M. Pessot et al., Opt. Lett. 14, 797 (1989).

[3] M. D. Perry and G. Mourou, Science 264, 917 (1994).

[4] M. D. Perry et al., Opt. Lett. 24, 160 (1999).

[5] G. Shvets et al., Phys. Rev. Lett. 81, 4879 (1998).

[6] V. M. Malkin, G. Shvets, and N. J. Fisch, Phys. Rev. Lett. 82, 4448 (1999).

[7] V. M. Malkin and N.J. Fisch, Phys. Plasmas 8, 4698 (2001).

[8] Y. A. Tsidulko et al., Phys. Rev. Lett. 88, 235004 (2002).

[9] N. J. Fisch and V. M. Malkin, Phys. Plasmas 10, 2056 (2003).

[10] M. Tabak et al., Phys. Plasmas 1, 1626 (1994).

[11] J. Dunn et al., Phys. Rev. Lett. 84, 4834 (2000).

[12] P. Sprangle et al., Phys. Rev. Lett. 69, 2200 (1992).

[13] Y. Ping et al., Phys. Rev. E 66, 046401 (2002).

[14] Y. Ping, I. Geltner, and S. Suckewer, Phys. Rev. E 67, 016401 (2003).

[15] Y. Ping et al., Phys. Rev. Lett. 92, 175007 (2004).

[16] A. Balakin et al., JETP Lett. 80, 15 (2004).

[17] M. Dreher et al., Phys. Rev. Lett. 93, 095001 (2004).

[18] N. Zhavoronkov et al., Opt. Lett. 26, 47 (2001).

[19] M. S. Hur et al., Phys. Plasmas 11, 5204 (2004).

[20] P. Mardahl et al., Phys. Lett. A 296, 109 (2002). 